\title{
Nonlinear Bending of the Block Spherical Reticulated Shells with Damage under the Transverse Load
}

\author{
Lei $\mathrm{Li}^{1}$, Sheng-dong $\mathrm{He}^{1}$, Ji-ping $\mathrm{Hao}^{2}$ \\ 1 ZhengZhou University of Aeronautics, Zhengzhou, China \\ ${ }^{2}$ Xi' an University of Architecture Technology, Xi'an, China
}

\begin{abstract}
Based on theory of Lemaitre's equivalent strain of the damage and with the damage of bars of the block reticulated spherical shell, the nonlinear bending problem of the block reticulated shells considering damage with rectangular bottom was studied under the boundary condition of moved fixed-edges based on the nonlinear deformation fundamental equations. The trigonometric series solution satisfies boundary condition of the deflection was derived firstly. The stress function was solved by substitution of the solution into the nonlinear compatibility equation and the nonlinear characteristic formula of the loads and the deflection was obtained by the energy equation. Then the critical point was given. The critical load was given by the characteristic curves. The nonlinear characteristic formula in the paper can be reference by the engineering designers. The effect of the damage of the structure can be considered.
\end{abstract}

\section{Problem of the block spherical reticulated shells with damage}

Based on theory of Lemaitre's equivalent strain of the damage and with the damage of bars of the block reticulated spherical shell, The control equations of the single layer three-dimensional shallow block spherical reticulated shell considering damage with equilateral triangle grid $^{[1,2]}$

$$
\begin{aligned}
& n_{1} L_{1}(w)(1-\omega)=L_{2}(w, \phi)+\kappa_{x} \frac{\partial^{2} \phi}{\partial y^{2}}+\kappa_{y} \frac{\partial^{2} \phi}{\partial x^{2}}+q \\
& \frac{n_{2}}{1-\omega} L_{1}(w)=L_{2}(w, \phi)+\kappa_{x} \frac{\partial^{2} \phi}{\partial y^{2}}+\kappa_{y} \frac{\partial^{2} \phi}{\partial x^{2}}+q
\end{aligned}
$$

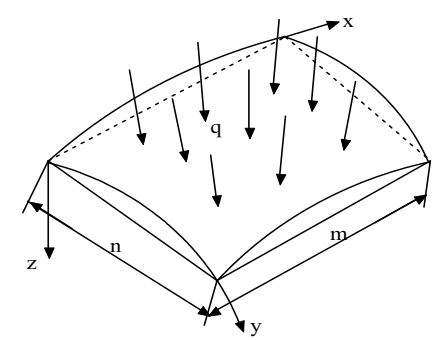

Fig 1. Free body schematic diagram of the block reticulated shell with damage
Where $L_{1}=\frac{\partial^{4}}{\partial x^{4}}+2 \frac{\partial^{4}}{\partial x^{2} \partial y^{2}}+\frac{\partial^{4}}{\partial y^{4}}$,

$$
L_{2}(w, \phi)=\frac{\partial^{2} w}{\partial x^{2}} \frac{\partial^{2} \phi}{\partial y^{2}}+\frac{\partial^{2} w}{\partial y^{2}} \frac{\partial^{2} \phi}{\partial x^{2}}-2 \frac{\partial^{2} \phi}{\partial x \partial y} \frac{\partial^{2} w}{\partial x \partial y}
$$

$n_{1}=\frac{3 \sqrt{3} E I}{4 a}, n_{2}=\frac{\sqrt{3} a}{2 E A}, \omega=\frac{s_{d}}{S}$, In order to simplify the

calculation, it is assumed

the structure damage is uniform distribution. $\omega$ is damage degree, $s_{d}$ is section area of the bars with damage, $s$ is section area of the bars without damage.

The boundary conditions of the block shell with the moved fixed-edges bottom:

When $\mathrm{x}=0, m, p_{x}=0, w=0, \frac{\partial w}{\partial x}=0$

When $\mathrm{y}=0, \mathrm{n}, p_{y}=0, w=0, \frac{\partial w}{\partial y}=0$

Noting: $\mathrm{p}_{\mathrm{x}}$ and $\mathrm{p}_{\mathrm{y}}$ the lateral unit width force of the shell (positive when compressed).

\section{SOLUTION OF THE PROBLEM}

Select the expression of the deflection

$$
w=\sum_{i} \sum_{j} f_{i j} \sin ^{2} i \frac{\pi}{m} x \sin ^{2} j \frac{\pi}{n} y
$$


Select the first approximation expression of the deflection of the shell

$$
w=f \sin ^{2} \frac{\pi}{m} x \sin ^{2} \frac{\pi}{n} y
$$

Where $f$ the symmetry centre deflection of the shell

Substituting (6) into deformation compatibility equation (2) we can obtain:

$$
\begin{aligned}
& \frac{n_{2}}{1-\omega} \phi=\frac{f^{2}}{32}\left[\frac{m^{2}}{n^{2}} \cos 2 \alpha x-\frac{n^{2}}{m^{2}} \cos 2 \beta y\right. \\
& -\frac{2 m^{2} n^{2}}{\left(m^{2}+n^{2}\right)^{2}} \cos 2 \alpha x \cos 2 \beta y-\frac{n^{2}}{8 m^{2}} \cos 4 \beta y \\
& +\frac{m^{2} n^{2}}{\left(m^{2}+4 n^{2}\right)^{2}} \cos 4 \alpha x \cos 2 \beta y \\
& \left.+\frac{m^{2} n^{2}}{\left(4 m^{2}+n^{2}\right)^{2}} \cos 2 \alpha x \cos 4 \beta y\right] \\
& +\frac{f \kappa_{x}}{16 \pi^{2}}\left[-n^{2} \cos 2 \beta y\right. \\
& \left.+\frac{m^{4} n^{2}}{\left(m^{2}+n^{2}\right)^{2}} \cos 2 \alpha x \cos 2 \beta y\right] \\
& +\frac{f \kappa_{y}}{16 \pi^{2}}\left[-m^{2} \cos 2 \alpha x\right. \\
& \left.+\frac{m^{2} n^{4}}{\left(m^{2}+n^{2}\right)^{2}} \cos 2 \alpha x \cos 2 \beta y\right] \\
& -\frac{n_{2}}{1-\omega}\left(\frac{p_{x} y^{2}}{2}+\frac{p_{y} x^{2}}{2}\right) \\
& +
\end{aligned}
$$

\section{Galerkin equation:}

$$
\int_{0}^{m} \int_{0}^{n} X \sin ^{2}\left(\alpha_{0} x\right) \sin ^{2}\left(\beta_{0} y\right) d x d y=0
$$

Where

$$
X=n_{1} L_{1}(w)(1-\omega)-L_{2}(w, \phi)-\kappa_{x} \frac{\partial^{2} \phi}{\partial y^{2}}-\kappa_{y} \frac{\partial^{2} \phi}{\partial x^{2}}-q
$$

Substituting (6) and (7) into equation (8), the following dimensionless quantities are introduced:

$$
\begin{aligned}
& \xi=\frac{f_{(t)}}{\beta}, \quad \lambda=\frac{m}{n}, \quad \kappa_{y}^{*}=\frac{\kappa_{y} m^{2}}{\beta}, \\
& \kappa_{x}^{*}=\frac{\kappa_{x} n^{2}}{\beta}, \quad p_{x}^{*}=\frac{n_{2} p_{x} n^{2}}{\beta^{2}}, \\
& p_{y}^{*}=\frac{n_{2} p_{y} m^{2}}{\beta^{2}}, \quad q^{*}=\frac{q m^{2} n^{2} n_{2}}{\beta^{3}}, \\
& \eta=\frac{9 \beta^{2}}{8 s}, \quad c^{*}=\frac{9 n_{2} m^{2} n^{2} c}{16 \beta^{2}},
\end{aligned}
$$

We can obtain:

$$
\begin{aligned}
& \lambda^{2} \kappa_{x}^{*} p_{x}^{*}+\frac{1}{\lambda^{2}} \kappa_{y}^{*} p_{y}^{*}-q^{*} \\
& +(1-\omega)\left\{\begin{array}{l}
\pi^{4} \eta \xi\left(3 \lambda^{2}+\frac{3}{\lambda^{2}}+2\right) \\
-\frac{3}{4} \pi^{2} \xi\left(p_{x}^{*}+p_{y}^{*}\right) \\
+\frac{\xi}{8}\left(\lambda^{2} \kappa_{x}^{* 2}+\frac{\kappa_{y}^{* 2}}{\lambda^{2}}\right)
\end{array}\right. \\
& +\frac{\xi}{16\left(\lambda+\frac{1}{\lambda}\right)^{2}}\left(\lambda^{2} \kappa_{x}^{*}+\frac{\kappa_{y}^{*}}{\lambda^{2}}\right) \\
& -\frac{\pi^{2} \xi^{2}}{16}\left[\begin{array}{c}
\frac{1}{\left(\lambda+\frac{1}{\lambda}\right)^{2}}\left(\begin{array}{c}
3 \lambda^{2} \kappa_{x}^{*} \\
+\frac{2 \kappa_{y}^{*}}{\lambda^{2}}
\end{array}\right) \\
+\kappa_{x}^{*}+3 \kappa_{y}^{*}+\frac{\kappa_{y}^{*}}{\lambda^{2}}
\end{array}\right] \\
& \left.+\frac{\pi^{4} \xi^{3}}{8}\left[\begin{array}{l}
\frac{1}{\left(\lambda+\frac{1}{\lambda}\right)^{2}}+\frac{1}{4\left(4 \lambda+\frac{1}{\lambda}\right)^{2}} \\
-\frac{7}{16 \lambda^{2}}+\frac{\lambda^{2}}{2}
\end{array}\right]\right\}=0
\end{aligned}
$$

When the edges of the block spherical reticulated shell considering damage with the bottom moved fixededges:

$$
p_{x}^{*}=0, \quad p_{y}^{*}=0, \text { we can obtain }
$$




$$
\begin{aligned}
& (1-\omega)\left\{\begin{array}{l}
\pi^{4} \eta \xi\left(3 \lambda^{2}+\frac{3}{\lambda^{2}}+2\right) \\
+\frac{\xi}{8}\left(\lambda^{2} \kappa_{x}^{* 2}+\frac{\kappa_{y}^{* 2}}{\lambda^{2}}\right) \\
+\frac{\xi}{16\left(\lambda+\frac{1}{\lambda}\right)^{2}}\left(\lambda^{2} \kappa_{x}^{*}+\frac{\kappa_{y}^{*}}{\lambda^{2}}\right)^{2}
\end{array}\right. \\
& -\frac{\pi^{2} \xi^{2}}{16}\left[\begin{array}{l}
\frac{1}{\left(\lambda+\frac{1}{\lambda}\right)^{2}}\left(3 \lambda^{2} \kappa_{x}^{*}+\frac{2 \kappa_{y}^{*}}{\lambda^{2}}\right) \\
+\kappa_{x}^{*}+3 \kappa_{y}^{*}+\frac{\kappa_{y}^{*}}{\lambda^{2}}
\end{array}\right] \\
& \left.+\frac{\pi^{4} \xi^{3}}{8}\left[\begin{array}{l}
\frac{1}{\left(\lambda+\frac{1}{\lambda}\right)^{2}}+\frac{1}{4\left(\lambda+\frac{4}{\lambda}\right)^{2}} \\
+\frac{1}{4\left(4 \lambda+\frac{1}{\lambda}\right)^{2}}-\frac{7}{16 \lambda^{2}}+\frac{\lambda^{2}}{2}
\end{array}\right]\right\}-q^{*}=0
\end{aligned}
$$

Based on the sufficient condition of the existence of the extreme value we can obtain:

at $\xi=\frac{-b-\sqrt{b^{2}-4 a c}}{2 a}, q^{\prime}$ maximum.

Where

$$
\begin{aligned}
& a=\frac{3 \pi^{4}}{8}\left[\frac{1}{\left(\lambda+\frac{1}{\lambda}\right)^{2}}\right. \\
& +\frac{1}{4\left(\lambda+\frac{4}{\lambda}\right)^{2}}+\frac{1}{4\left(4 \lambda+\frac{1}{\lambda}\right)^{2}} \\
& \left.-\frac{7}{16 \lambda^{2}}+\frac{\lambda^{2}}{2}\right]=0
\end{aligned}
$$$$
b=-\frac{\pi^{2}}{8}\left[\frac { 1 } { ( \lambda + \frac { 1 } { \lambda } ) ^ { 2 } } \left[3 \lambda^{2} \kappa_{x}^{*}\right.\right.
$$$$
\left.\left.+\frac{2 \kappa_{y}^{*}}{\lambda^{2}}\right]+\kappa_{x}^{*}+3 \kappa_{y}^{*}+\frac{\kappa_{y}^{*}}{\lambda^{2}}\right]
$$

$$
\begin{aligned}
& c=\pi^{4} \eta\left(3 \lambda^{2}+\frac{3}{\lambda^{2}}+2\right) \\
& +\frac{1}{8}\left(\lambda^{2} \kappa_{x}^{* 2}+\frac{\kappa_{y}^{* 2}}{\lambda^{2}}\right) \\
& +\frac{1}{16\left(\lambda+\frac{1}{\lambda}\right)^{2}}\left(\lambda^{2} \kappa_{x}^{*}+\frac{\kappa_{y}^{*}}{\lambda^{2}}\right)^{2}
\end{aligned}
$$

The characteristic curves were given in the following:

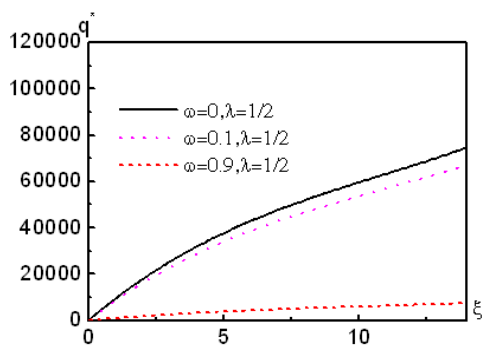

(a)

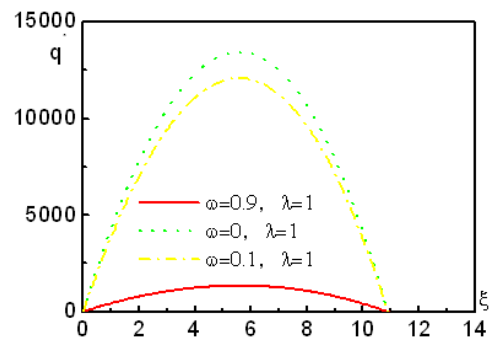

(b)

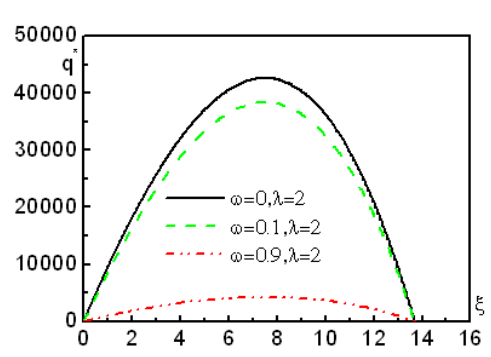

(c)

Fig. 2 Characteristic curve of the load and the deflection

$$
\kappa_{x}^{*}=\kappa_{y}^{*}=100, \quad \eta=2
$$

From the fig. 2 we can obtain when $\lambda=\frac{1}{2}$, with being the damage degree larger, is the deflection larger; when $\lambda=1$ and $\lambda=2$, when the damage is larger, the load to the maximum the curves declines quickly, the structure is instability. The shells with damage the easier instability.

\section{Conlusion}

The boundary value problem of the block shells with damage is much greater difficult than the problem of the shells with circle bottom ${ }^{[3,4,5]}$, the different critical 
load of different rectangular block shells with damage can be given based on the value of the edge length ratio. It is easier instability for the reticulated shells with the damage.

\section{Acknowledgement}

I wish to express my gratitude to NSFC(U140454) and Scientical and Technical project of Henan province (142300410040) support. I appreciate my research supervisor helping.

\section{References}

1. Zu-Yan Shen, Yang-ji Chen. Grid and Reticulated shells, Tongji Press, Shanghai: (1998).

2. Xin-zhi Wang, Cong-xing Liang, Ming-jun Han. Applied Mathematics and Mechanics, 28(2) (2007).

3. Xinzhi Wang, Yonggang Zhao, Kaiyuan Yeh. Applied Mathematics and Mechanics,19(10)(1998).

4. Xin-zhi Wang. Journal Of Gansu University Of Technology. 1(1980)

5. Xin-zhi Wang, Lin Li, Gang Wang. Engineering Mechanics, 25(10)(2008).

6. Lei Li, Yi Huang. Journal of Dynamics and Control. 7(4) (2009).

7. J. Lemaitre. Continuum damage mechanics. Berlin: Springer- verlag(1992).

8. Yun Wang, Ji-ping Hao. Geometrical Topology of Elastic Damage Theory. Engineering Mechanics. 25(5) (2008) 